\title{
Experience of significant hemodynamic instability that occurred during excisional biopsy in a patient with unrecognized bronchial carcinoid tumor
}

\author{
Sangil Lee, Kang Hun Lee, Ji Yeon Kim, Won Joo Choe, and Jung Won Kim
}

Department of Anesthesiology and Pain Medicine, Ilsan Paik Hospital, Inje University School of Medicine, Goyang, Korea

Bronchial carcinoid tumor is rare, accounting for $1-2 \%$ of all lung tumors [1]. If accurate diagnosis of a carcinoid tumor is made before an operation, and appropriate medical treatment is given, anesthetic management may improve [2]. However, patients without symptoms may not receive a diagnosis for the disease. As a result, patients who are not properly diagnosed could experience a life-threatening event such as carcinoid crisis in response to the anesthesia and surgery they are given [2]. We present a patient who experienced significant hemodynamic instability during excisional biopsy of a solitary pulmonary nodule suspected to be lung cancer.

A 55-year-old male presented to the hospital, complaining of a dry cough that had continued intermittently for two months. He had no other underlying diseases, but had a history of smoking for about 20 years. A solitary pulmonary nodule was found on chest radiograph, and the chest computed tomography showed a well-enhancing mass lesion with calcification in the right middle lobe bronchus. A polypoid mass was detected on bronchoscopic examination and bronchial washing cytology was performed. The results of the washing cytology were nonspecific, however, so an excisional biopsy was planned to rule out lung cancer.

The patient's preoperative vital signs were blood pressure $115 / 75 \mathrm{mmHg}$, heart rate 90 beats/min, and oxygen saturation $100 \%$. An epidural catheter (Perifix ${ }^{\circledR}$ FX Springwound, B. Braun, USA) was placed between T6 and T7 for postoperative pain control. Endotracheal intubation was performed for one-lung ventilation utilizing a 35Fr Silicon Double-Lumen
Endobronchial Tube (Silbroncho ${ }^{\circledR}$, Fuji, Tokyo, Japan). After induction, the patient's systolic and diastolic blood pressure were $100-120 \mathrm{mmHg}$ and $60-80 \mathrm{mmHg}$ and his heart rate and oxygen saturation were $80-90$ beats/min and $100 \%$, respectively. Fifteen minutes after starting the operation, the pulse suddenly spiked to 160 beats/min, the blood pressure decreased to $65 / 35 \mathrm{mmHg}$, and atrial fibrillation was observed on the ECG. The pulse rate and the systolic and diastolic blood pressure radically changed to $100-150$ beats $/ \mathrm{min}, 60-130$ $\mathrm{mmHg}$, and 35-80 $\mathrm{mmHg}$, respectively, after the event (Fig. 1).

A loading dose of $10 \mathrm{mg}$ diltiazem $\left(\operatorname{Herben}^{\circledR}, \mathrm{CJ}\right.$, Seoul,

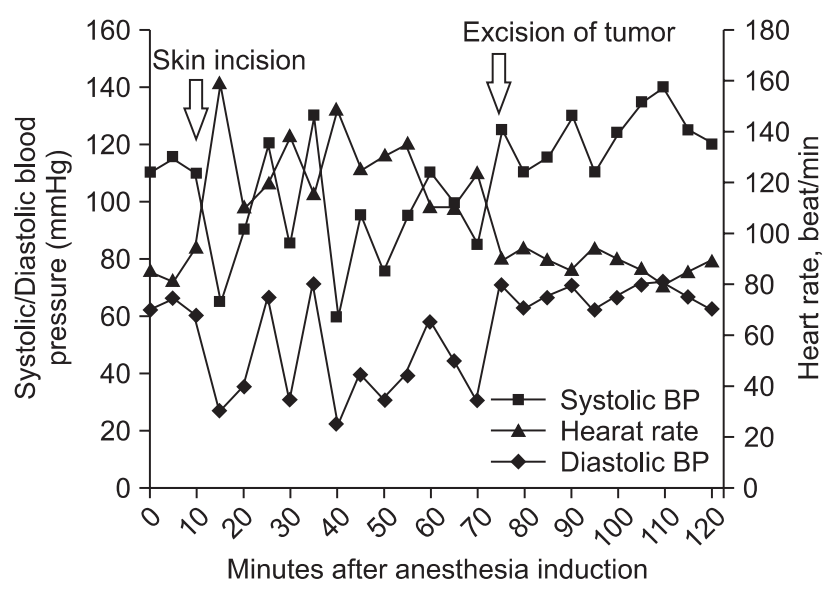

Fig. 1. Changes in systolic and diastolic blood pressure (BP) and heart rate during surgery.

Corresponding author: Sangil Lee, M.D., Department of Anesthesiology and Pain Medicine, Ilsan Paik Hospital, Inje University School of Medicine, 2240, Daehwa-dong, Ilsanseo-gu, Goyang 411-706, Korea. Tel: 82-31-910-7160, Fax: 82-31-910-7183, E-mail: knighder@nate.com (c) This is an open-access article distributed under the terms of the Creative Commons Attribution Non-Commercial License (http:// creativecommons.org/licenses/by-nc/3.0/), which permits unrestricted non-commercial use, distribution, and reproduction in any medium, provided the original work is properly cited. 
Korea) was injected intravenously, and the infusion was continued at a rate of $3 \mu \mathrm{g} / \mathrm{kg} / \mathrm{min}$ to control the patient's heart rate. Dobutamine (Dobutamine $\mathrm{HCl}$ Injection ${ }^{\circledR}$, Hana, Seoul, Korea) was also injected intravenously, at a rate of $10 \mu \mathrm{g} / \mathrm{kg} /$ min, due to suspicion of hypotension caused by low cardiac output. Following injection of the medication, the patient's pulse rate dropped to $100-120$ beats/min and the atrial fibrillation disappeared, but radical blood pressure changes continued. $100 \mu \mathrm{g}$ of fentanyl and $4 \mathrm{cc}$ of normal saline solution were injected via the epidural catheter, as the patient was thought to be sensitive to surgical pain. However, the pulse remained the same, and the blood pressure decreased to $75 / 40$ $\mathrm{mmHg}$. As there were no underlying diseases that could explain the patient's unstable vital signs, we requested that the surgeon conclude the operation as rapidly as possible.

The mass lesion was removed one hour after the surgery began. After the removal, the systolic and diastolic blood pressure stabilized at $100-140 \mathrm{mmHg}$ and $60-70 \mathrm{mmHg}$, respectively (Fig. 1). Histopathologic examination confirmed the diagnosis of bronchial carcinoid tumor.

Perioperative anesthetic management during the surgical removal of carcinoid tumors aims to avoid triggering factors that stimulate the secretion of bioactive materials. However, anesthesiologists should be prepared for the situation that might arise if carcinoid crisis occurs despite these efforts. Octreotide (Sandostatin ${ }^{\circledR}$, Novartis) plays an essential role in both treatment of carcinoid tumors prior to surgical removal and management of carcinoid crisis during surgery. Octreotide is a synthetic analog of somatostatin. It binds tightly to the type 2 somatostatin receptor on tumor cells. It prevents the tumor from releasing a mediator and counteracts the activities of the circulating peptides by inhibiting either phosphatidylinositol or adenylate cyclase [3].

Secretion of bioactive materials from carcinoid tumors during surgery may result as a response to endotracheal intubation, inadequate pain management, or hypotension. In our case, it was hypothesized that the carcinoid crisis might have been triggered by the surgeon's manipulation of the tumor. When unstable hemodynamic conditions occur, it is important for the operating surgeon to cease the manipulation of the tumor until the vital signs are stabilized [3]. Thus we should have asked the surgeon to stop the operation at the onset of the carcinoid crisis and administered an experimental IV injection of octreotide. However, octreotide is not commonly used in the field of anesthesiology, so it cannot be prepared quickly. We therefore need to take into consideration the fact that there are restrictions on its potential use at appropriate times when the need for it arises unexpectedly.

We had implanted a thoracic epidural catheter in order to control postoperative pain, but a sympathetic block could increase the severity of hypotension. Severe hypotension may cause carcinoid crisis by stimulating the secretion of tumor mediators. In this case, we estimated that hypotension caused by the injection of fentanyl via the epidural catheter stimulates the secretion of tumor mediators, which result in the high risk of carcinoid crisis. Of course, there are cases in which patients with carcinoid symptoms have undergone successful epidural anesthesia during non-carcinoid surgery [4]. However, given the above-mentioned risk factors, it is necessary to closely monitor cardiovascular parameters and the levels of the regional block when placing an epidural catheter for controlling pain in patients with possible carcinoid symptoms [5].

Carcinoid tumors present anesthetic challenges due to their particular nature and various clinical patterns. The pathophysiology of carcinoid is well known, but intraoperative carcinoid events are difficult to predict [3]. Presently, octreotide plays a pivotal role both in managing carcinoid symptoms before surgery and in controlling acute symptoms of carcinoid crisis during surgery. For appropriate anesthetic management of carcinoid patients, therefore, anesthesiologists should have full knowledge of the pathophysiology of the disease as well as of the characteristics of octreotide and its proper use. They also need to consider the possibility of carcinoid tumor in all surgical patients with lung tumors who have not received an accurate pathological diagnosis.

\section{References}

1. Jeung MY, Gasser B, Gangi A, Charneau D, Ducroq X, Kessler R, et al. Bronchial carcinoid tumors of the thorax: spectrum of radiologic findings. Radiographics 2002; 22: 351-65.

2. Hudcova J, Schumann R. Undiagnosed catecholamine-secreting paraganglioma and coexisting carcinoid in a patient with $\mathrm{MH}$ susceptibility: an unusual anesthetic challenge. J Anesth 2007; 21 : 80-2.

3. Mancuso K, Kaye AD, Boudreaux JP, Fox CJ, Lang P, Kalarickal $\mathrm{PL}$, et al. Carcinoid syndrome and perioperative anesthetic considerations. J Clin Anesth 2011; 23: 329-41.

4. Monteith K, Roaseg OP. Epidural anaesthesia for transurethral resection of the prostate in a patient with carcinoid syndrome. Can J Anaesth $1990 ; 37: 349-52$.

5. Dierdorf SF. Carcinoid tumor and carcinoid syndrome. Curr Opin Anaesthesiol 2003; 16: 343-7. 